so many living germs that if uniformly diffused through the pint of air, not a thimbleful could be taken without containing several. These germs, placed under favourable circunistances, would soon grow large enough to be detected without any difficulty.

LIONEL S. BEALE

The Source of Solar Energy

IT is, I think, rather unfortunate that Mr. Proctor, in his recent work entitled "More Worids than One," should have re-adyocated the earlier and now discarded views of Sir W. Thoinson concerning the source of solar heat or energy by meleric percussion. 'That theory, lowever ingenions as advanced by the physicist, is surely hardly one to be admitted by the astronomer. Nothing less than an intense desire or necessity for finding some solution to the problem, whence or how the solar heat is maintained, conld have encouraged scientific men seriously to advance or support so plausible and unsatisfactory a doctrine, or one, when exanined, so little supported by what we really know either of meteors or of nature's laws. Having given much attention to meteoric astronomy, may I be permitted briefly to state what I hold are 'serious and practical objections to the validity of the meteoric or dynamical theory as applied to the conservation of solar heat and energy.

r. Because meteors and aerolites are known to impinge and strike the earth in her orbit, ergo, as I understand Mr. Proctor; numbers infinitely greater must no doubt be constantly rushing into the sun, as a body at once far larger, and much nearer to myriads of such bodies than the earth herself; but which, at a moly smaller distance, are more likely to be diawn into the sun. Now, all that we really do know about meterrs amounts to this, that by far the greater number of shooting stars visible in our atmosphere, in size no larger than a bean, and really separated from cach other by thousands of miles, belong to fixed and definite systems or rings, having fixed radiant points for certain epochs or periods, showing clearly that these bodies are revolving round the sun, in courses as true and regular as the planets themselves, and are no more eddying or rushing into the sun, inerely because they are 50 insignificant, than is the earth herself. Having projected upon celestial clarts the apparent courses or tracks of nearly 5,c00 meteors, observed during every part of the year, I feel I am justified in stating that not more than seven or eight per cent, of the shooting stars observed on any clear night throughout the year, are sporadic, or do not belong to meteor systems at present known to us. More than one hundred meteor systems are now recognised, several of which appear most certainly to be connected with known comets; and from a paper I have just received from Professor Schiaparelli, of Milan, it would appear that the approximate average perilution distance for 44 of these meteor systems is not less than $0 \%$, the earth's distance from the sun being I $\circ$; whilst of these 44 systems, only 4 , or about ro per cent. have their perihelion distance under $0 \cdot 1$, that is, approach the sun nearer than nine millions of miles! Now, it is pretty well admitted that meteors are intimately connected with comet systems, yet out of some 200 comets, the elements of whose orbits have been calculated with tolerable precision, only 5 per cent. have their perihclion distance under $\mathrm{O}$ I. The saine argument holds good also for planets, whose numbers also diminish after a certain considerable mean distance from the sun. Are these facts, then, in accordance with the notion that meteoric bodies either increase in number as we approach the sun, or that meteors are so constantly losing their senses, or sense of gravity, as to be ever rushing into or against the sun? I might almost ask, do any meteors rush or fall into the sun? Is it probable that the mass of all "the countless myriads of meteors" in the solar system exceeds that of a single planet? whether that of Mercury or Jupiter does not much signify. When we take into consideration the gigantic amount of meteoric deposits required to maintain the solar heat for hundreds of millions of years, in the meteoric theory, surely the supply of meteors would long since have been exbausted, were the supply at least confined merely to the meteors under a mean distance of $O^{\prime} \mathrm{I}$ belonging to our own solar system! The argument, to legin with, is in a great degree fallacious, e.g, because meteors frequently strike the earth, they must, it is argued, strike the sun in vastly creater numbers, and with far greater velocities. But it is forsrotten that the meteors themselves, like the earth, are revolving round the sun as a common centre, in regular orbits, and only by accident, as it were, come into mutual collision, just as the tail of a comet might pass through the system of Jupiter and his satellites; while to the end of time neither the earth nor the meteors need necessarily come into contact with the sun.
2. But it is not inerely meteors belonging to the solar system which are taxed to provide fuel for our sun; space itself may be filled with meteors rearly to impinge upon the sun. The arguments against this are : (I) judging from analogy as well as from facts, comparatively few meteors are sforadic, consequently the majority cannot belong to stellar space, but to our own system ; (2) granting that space itself is really more or less filled with meteors, these would not necessarily rush straight into the sun, unless, as would very unlikely be the case, they had no proper motion of their own. They might be drawn into or enter our system, it is true, but, according to Schiaparelli, only to circu'ate like comets in definite orlsits.

3. The zodiacal light is another victim to the emergencies of the meteoric theory of solar energy. Whethre composed of myriads of small meteors, or mexely a nebulous appendage, or atmospheric emanation belonging to the sun, is it credible that for hundreds of millions of years there could, physically speaking, be sufficient material in the zodiacal light to maintain the sun's heat and supply all the fuel required? Has it ever yet been proved that the entire mass of matter constituting the zodiacal light, is either composed of 'matter in a solid state, or, if it were, that its mass would be equal to that of our own earth? If composed of separate meteors, are they not each individually revolving round the sun, rather than occupied in being gradually drawn into it as a vortex? *

Of course I do not say that meteors are nevier drawn into the sun, or that they may not occasionally and by accident enter the solar atmosphere; I have merely endeavoured to show that, from what we really do know about neteors and the laws of nature, it is highly improbable that our sun could derive, in sufficient quantity, a needful supply of fucl from meteoric sources. The comet of 1843 , which approached the sun within 550,000 miles, was not sensibly deflected from its course; it is just possible that so small a thing as an aerrolite might at that clistance have been drawn into the sun; but is it not also possible, from what we know of comet and meteor systems, it may be wisely ordained that the smaller bodies of our solar system, such as meteors, do not as a rule approach the sun too closely; and they probably do not, if their perihelia distances are rarely under 10,000,000 of miles?

Aërolites are doubtless of larger size and weight than shooting stars, and, as far as is yet known, not so regular in their appearance as shooting stars; but even with that class of phenomena, we notice a certain degree of periodicity in maxima and minima for certain times of the year, tending to show that they also may be stubject to regular law's, and not fall so frequently or promiscuously upon the sun's surface as has been sometimes supposed. If they do not fall in vastly greater numbers, area for area, upon the sun than they do upon our earth, certainly the dynamical effect would be very minute! I may here also observe that $\epsilon$ ven these bodies generally fall to the earth without being consumed, and with a very moderate velocity ; their original cosmical velocity having been lost before reaching the surface of the earth. In the case of an aërolite falling upon the sun's surface, its original velocity may similarly have been gradually checked in its passage through the solar atmosphere, and a considerable amount therefore of the calculated mechanical effect lost. Small meteors would probably be consumed thousands of miles from the real body of the sun, seeing that the sun's inflamed atmosphere is now known to extend at times some 50,000 miles. It might almost be a question whether the sun's proper heat may not even be greater than that caused by the simple friction of a meteor through the solar atmosphere

I merely allude to these minor matters, however, in order to point out some of the numerous uncertainties and difficulties connected with this meteoric or mechanical theory of the origin and conservation of solar heat, in addition to those already alluded to, bearing more especially upon the astronomical bearings of the question. For the present it must still remain a mystery, whence or how the solar heat is maintained, or to what extent really wasted.

Prestwich, Manchester, July I I

Robert P. Grec

\section{Choice of a Microscope}

Wirt all respect to the judgment of my friend $\mathrm{Mr}$. Ray Lankester, I should like to be allowed to oppose a few of the statements made by him in his remarks on the Choice of a

* We beg to refer our readers to Jones* and Liais' observations of the Zodiacal Light. They certainly have not received the attention in this country that they deserve, $-\mathrm{ED}$. 